\title{
Evaluación de la microcirculación sublingual en un paciente en shock séptico refractario tratado con hemofiltración de alto volumen
}

\author{
Carolina Ruiz B, Alejandro Bruhn C, \\ G lenn Hernández $P$, Max Andresen $H$.
}

\section{Evaluation of sublingual microcirculation in septic shock. Report of one patient treated with high volume hemofiltration}

\begin{abstract}
Microcirculation is severely compromised in sepsis, with a reduction of capillary density and flow impairment. These alterations have important prognostic implications, being more severe in non-survivors to septic shock. Today microcirculation may be assessed bedside, non-invasively, using polarized light videomicroscopy, a technique known as SDF (side dark field). We report a 54 year-old man with an extramembranous nephropathy, that developed a necrotizing fascitis associated to septic shock, in whom microcirculation was periodically assessed during his management. The patient was treated with fluids, vasoactive drugs, antibiotics and was operated for exploration and debridement. As the patient persisted in refractory shock despite treatment, highvolume hemofiltration was started. Before hemofiltration the patient had severe microcirculatory alterations that improved during and after the procedure. Physiologic endpoints of high-volume hemofiltration in septic shock remain unknown, but it has the capacity to clear inflammatory mediators. Since microcirculatory alterations are in part secondary to these mediators, their removal is beneficial. Like other authors, we found no relation between microcirculation and other haemodynamic and perfusion variables (Rev Méd Chile 2008; 136: 1175-8).
\end{abstract}

(Key w ords: Hemofiltration; Microcirculation; Shock, septic)

\begin{abstract}
Recibido el 14 de noviembre, 2007. Aceptado el 22 de enero, 2008.
Departamento de Medicina Intensiva, Facultad de Medicina, Pontificia Universidad Católica de Chile. Santiago de Chile.
\end{abstract}

L a microcirculación es la red de capilares, arteriolas y vénulas, menores a 100 micrómetros de diámetro, cuya función es transportar oxígeno y nutrientes a las células. Esta se compromete gravemente en la sepsis, debido a la gran liberación de mediadores inflamatorios y a la

Correspondencia a: Dra. Carolina Ruiz B. Departamento de Medicina Intensiva, Facultad de Medicina, Pontificia Universidad Católica de Chile. Marcoleta 367, Santiago, Chile. Teléfono: 3543746. Fax: 6641329. E mail: crbchile@yahoo.com activación de la coagulación, produciéndose importantes alteraciones tanto en el endotelio como en las células circulantes ${ }^{1}$, con la consiguiente disminución de la densidad capilar y alteraciones en el flujo microcirculatorio, coexistiendo áreas de flujo interrumpido o intermitente, con otras en que se mantiene un flujo continuo ${ }^{2}$. Lo anterior provoca hipoxia tisular, aun cuando el transporte de oxígeno global (DO2) esté aumentado, con el consiguiente daño celular y disfunción de órganos $^{3}$. Estas alteraciones tienen importantes implicancias pronósticas, siendo más severas en los 
pacientes que fallecen en shock séptico (SS) ${ }^{2}$. Asimismo, la persistencia de estas alteraciones una vez resuelto el shock se asocia a la presencia de más disfunciones orgánicas y riesgo de muerte ${ }^{4}$, por lo que incluso algunos autores han planteado que el compromiso de la microcirculación seńa el motor del síndrome de disfunción orgánica múltiple (SDOM) ${ }^{1}$.

Aún no está claro cómo se relacionan las variables macrohemodinámicas con las alteraciones microcirculatorias en la sepsis, con resultados controvertidos hasta el momento ${ }^{2,4,5}$. Tampoco está clara la relación entre las alteraciones microcirculatorias a nivel sublingual y el compromiso microcirculatorio en otros territorios. La mucosa sublingual comparte el mismo onigen embriológico con la mucosa del tubo digestivo, por lo que se ha planteado que existińa una comelación entre ambas, pero los resultados también han sido contradictorios ${ }^{6,7}$.

La videomicroscopia de luz polanizada, conocida como SDF (side dark field), es una técnica que permite evaluar la microcirculación en forma no invasiva y a la cabecera del paciente. Consiste en un video-microscopio portátil (Microscan ${ }^{\circledR}$, Microvision Medical, Amsterdam, Holanda), que emite una luz polarizada de $550 \mathrm{~nm}$. Esta luz es absorbida por la hemoglobina y reflejada por el resto de las estructuras, por lo que en la imagen obtenida (imagen reflejada) se visualizan por contraste los glóbulos rojos fluyendo en la microcirculación. Estas imágenes de video son grabadas y posteriormente analizadas, existiendo varios índices para evaluar las alteraciones microcirculatorias ${ }^{8}$, entre los que destaca el Índice de Flujo Microvascular (Microvascular Flow Index, MFI), que es un indicador semicuantitativo que caracteriza el patrón de flujo predominante en 4 categonáas ( 0 sin flujo, 1 flujo intermitente, 2 flujo lento y 3 flujo normal) ${ }^{9}$. La limitante de esta técnica es que sólo permite evaluar superficies mucosas, por lo que la región más estudiada es el área sublingual.

A continuación se presenta el caso clínico de un paciente en SS refractario, tratado con hemofiltración de alto volumen (HFAV) como terapia de rescate hemodinámico ${ }^{10}$, con evaluación periódica de la microcirculación durante el procedimiento.

\section{CASO CĹNICO}

Paciente de 54 años con antecedentes de un síndrome nefrótico secundario a una nefropatía extramembrano- sa (proteinuria masiva) de 3 meses de evolución, en tratamiento con corticoides y con función renal conservada. Ingresó el 22/12/06 a la Unidad de Cuidados Intensivos (UCI) por un shock séptico secundario a una fasceítis necrotizante de la piema derecha. Al ingreso, el paciente ya presentaba una SDOM, con compromiso hemodinámico, respiratorio (injuria pulmonar aguda), renal, neurológico y de coagulación, con un score de APACHE II de 24. La reanimación inicial se realizó de acuerdo al protocolo de manejo de shock séptico de nuestra Unidad ${ }^{10}$ y a las recomendaciones de la Campaña de Sobrevida de la Sepsis ${ }^{11}$. Se conectó a ventilación mecánica (VM), se reanimó con fluidos $(3.300 \mathrm{ml}$ en las primeras $6 \mathrm{~h}$ entre cristaloides y coloides, manteniendo POAP mayores a $15 \mathrm{mmHg}$ ) y drogas vasoactivas, se le administraron corticoides (hidrocortisona $100 \mathrm{mg} \mathrm{c} / 8$ h) y se iniciaron antibióticos (vancomicina e imipenem por antecedentes de una hospitalización reciente). Fue llevado a pabellón donde se realizó un aseo quinúrgico con fasciotomía de la piema derecha. Evolucionó con un shock refractario (patrón hemodinámico hiperdinámico con índice cardiaco mantenido sobre $5 \mathrm{~L} /$ $\mathrm{min} / \mathrm{m}^{2}$ durante las primeras $12 \mathrm{~h}$ de evolución), con altos requerimientos de vasopresores (noradrenalina $0,56 \mathrm{mcg} / \mathrm{kg} / \mathrm{min}$ ) y marcada hipoperfusión (lactato $4,7 \mathrm{mmol} / \mathrm{l}$ ), por lo que se inició $\mathrm{HFAV}$, recibiendo un pulso de $24 \mathrm{~h}$ (UF de $100 \mathrm{ml} / \mathrm{kg} / \mathrm{h}$, flujo sanguíneo de $250 \mathrm{ml} / \mathrm{min}$, hemofiltro Ultraflux AV $600^{\circledR}$ ). Durante el procedimiento, mantuvo una mala evolución, con aumento de los requerimientos de vasoactivos (noradrenalina y adrenalina) y mayor hipoperfusión.

La microcirculación se evaluó previo al inicio de la HFAV (a las $10 \mathrm{~h}$ de ingreso a la UCI), a las 12 de conexión y a las 6 h postsuspensión (Tabla 1). En la primera evaluación presentaba severas alteraciones de la microcirculación, con un MFI de 1,15 (Figura 1), evolucionando posteriormente con mejoría de estas alteraciones (Figura 2), lo que no se correlacionó con los otros parámetros hemodinámicos ni de perfusión.

A los 4 días de evolución se suspendieron las drogas vasoactivas, y a los 10 días, tras varios aseos quirúrgicos, se le realizó una amputación infracondílea. Posteriormente evolucionó con recuperación de sus disfunciones salvo la falla renal, por lo que requirió apoyo dialítico, pero presentó varias intercurrencias infecciosas (neumonía nosocomial y sepsis por catéter venoso central). El paciente egresó de la UCI a principios de febrero de 2007, siendo dado 
Tabla 1. Variables hemodinámicas, de perfusión y M FI durante la H FAV

\begin{tabular}{|lccc|}
\hline Parámetro & Basal & $12 \mathrm{~h}$ de HFAV & 6 h post HFAV \\
\hline PAM $(\mathrm{mm} \mathrm{Hg})$ & 67 & 75 & 75 \\
FC $(\mathrm{LPM})$ & 121 & 110 & 115 \\
$\mathrm{IC}\left(\mathrm{L} / \mathrm{min} / \mathrm{m}^{2}\right)$ & 5,5 & 3,2 & 2,8 \\
POAP $(\mathrm{mm} \mathrm{Hg})$ & 26 & 21 & 15 \\
$\mathrm{Hcto}(\%)$ & 25,5 & 34,4 & 34 \\
Lactato $(\mathrm{mmol} / \mathrm{L})$ & 4,7 & 6 & 8 \\
SvO $(\%)$ & 79 & 77 & 79 \\
$\mathrm{SOFA}$ & 10 & 13 & 13 \\
$\mathrm{NA}(\mathrm{mcg} / \mathrm{kg} / \mathrm{min})$ & 0,56 & 1,2 & 0,9 \\
MFI & 1,15 & 2,2 & 2,3 \\
\hline
\end{tabular}

FC (frecuencia cardiaca), LPM (latidos por minuto), PAM (presión arterial media), IC (índice cardiaco), POAP (presión de oclusión de arteria pulmonar), Hcto (hematocrito), SOFA (Sepsis-related Organ Failure Assessment), $\mathrm{SvO}^{2}$ (saturación venosa mixta de oxígeno), NA (noradrenalina), MFI (índice de flujo microcirculatorio), HFAV (hemofiltración de alto volumen).

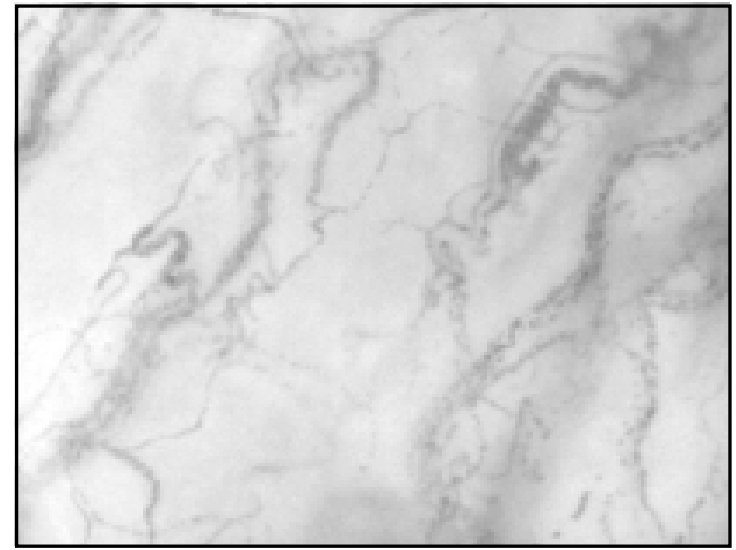

Figura 1. Microcirculación sublingual en condiciones basales. Microcirculación severamente alterada con MFI (índice de flujo microcirculatorio) 1,15.

de alta el 18/2/07. Actualmente se mantiene en control en el policlínico de nefrología de nuestro hospital, con hemodiálisis trisemanal.

\section{DisCUSIÓN}

No existen terapias validadas para optimizar la microcirculación en pacientes sépticos, tampoco se ha evaluado si la restauración del flujo microcirculatorio podńa ser una meta de reanimación, pero sí se ha

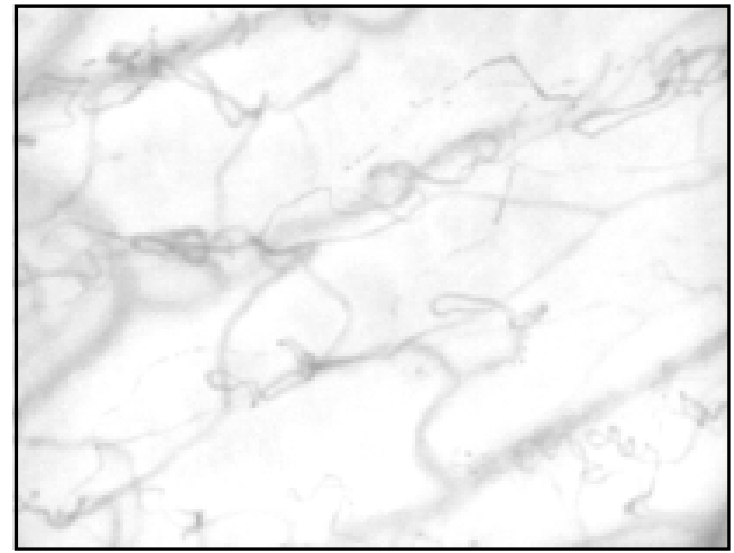

Figura 2. Microcirculación sublingual a las $12 \mathrm{~h}$ de HFAV. Mejonía de la microcirculación luego de $12 \mathrm{~h}$ de HFAV (hemofiltración de alto volumen). MFI (índice de flujo microcirculatorio) 2,2 .

evaluado el impacto de algunos tratamientos habituales del SS sobre la microcirculación. Los vasopresores mejorańan el flujo a nivel de los grandes vasos, pero no está claro su efecto a nivel de la microcirculación, lo que sí se ha demostrado es que los vasodilatadores (acetilcolina tópica y nitroglicenina endovenosa en bolo) mejoranian notablemente las alteraciones microcirculatorias ${ }^{2,12}$. Por esto, se ha planteado que un manejo con metas de presión no siempre es adecuado para reclutar a la microcirculación, y en forma secundaria mejorar la perfusión tisular ${ }^{1}$. También se 
ha estudiado el efecto de la dobutamina, encontrando una mejonía de las alteraciones microcirculatorias independiente del efecto de ésta sobre el índice cardiaco, la presión arterial u otras variables macrohemodinámicas, por lo que los autores de este estudio plantean una posible acción vasodilatadora de la dobutamina sobre las arteriolas, para explicar la mejońa en la microcirculación ${ }^{13}$.

Todavía no está claro a qué nivel actúa la HFAV en el SS, pero se cree que podńa remover mediadores proinflamatorios $^{14}$, por lo que algunos grupos, entre ellos el nuestro, ha comenzado a utilizarla como terapia de rescate hemodinámico, incluso en ausencia de insuficiencia renal, con resultados muy alentadores $^{10}$. Es esperable que si parte de las alteraciones microcirculatorias son secundarias a estos mediadores, al utilizar una técnica que en teonía los remueve, la microcirculación podńa mejorar.

Esta es la primera expeniencia que observa los posibles efectos de la HFAV sobre la microcirculación sublingual en un paciente en SS, destacando su mejonía en paralelo con la HFAV. Llama la atención que la mejonía de la microcirculación no tuviera relación con otras variables macrohemodinámicas y

\section{REFERENCIAS}

1. INCE C. The microcinculation is the motor of sepsis. Critical Care 2005; 9 (suppl 4): S13-19.

2. De Backer D, Creteur J, Preiser JC, Dubois MJ, Vincent JL Microvascular blood flow is altered in patients with sepsis. Am J Respir Crit Care Med 2002; 166: 98-104.

3. Euns C, JagGer J, Sharpe M. The microcirculation as a functional system. Critical Care 2005; 9 (suppl 4): S3-18.

4. Sakr Y, Dubois MJ, De Backer D, Creteur J, Vincent JL. Persistent microcirculatory alterations are associated with organ failure and death in patients with septic shock. Crit Care Med 2004; 32: 1825-31.

5. Trzeciak S, Delunger R, PariLo Je, Guglelmi M, Bajaj $\mathrm{J}$, ABATE N et al. Early microcirculatory perfusion deragements in patiens with severe sepsis and sectic shock: relationship to hemodynamics, oxygen transport, and survival. Ann Emerg Med 2007; 49: 88-98.

6. Creteur J, De Backer D, Sakr Y. Sublingual PCO2 monitoring in patients with septic shock. [Abstr] Crit Care Med 2003; 30:A19

7. Boerma C, Van Der Voort P, Spronk P, Ince C. Relationship between sublingual and intestinal microcirculatory perfusion in patients with abdominal sepsis. Crit Care Med 2007; 35: 1055-60.

8. De Backer D, Holennberg S, Boerma C, Goedhart P, de perfusión, pero como se señaló anteriomente, todavía no está clara la relación de la microcirculación con estas variables. De Backer y Sakr no encontraron una comelación entre las variables macrohemodinámicas y de perfusión y la microcirculación sublingual2,4; por el contrario, Trzeciak encontró recientemente una correlación adecuada del MFI con la presión arterial y los requerimientos de vasopresores dentro de las primeras $6 \mathrm{~h}$ de evolución del SS, no así en evaluaciones posteriores ${ }^{5}$. Destaca la evolución favorable de este paciente a pesar de la gravedad inicial y mala evolución durante las primeras 48 h, lo que es concordante con la mejonía que presentó a nivel de la microcirculación.

En la actualidad existe bastante evidencia del rol de la microcirculación en el shock séptico, de cómo sus alteraciones están relacionadas con el desarrollo de disfunciones, así como del valor pronóstico que tienen. Es por esto que nos parece que la evaluación de la microcirculación con la videomicroscopia de luz polarizada podría ayudar a evaluar el impacto de nuevas terapias para el shock séptico, incluso con el potencial de transformarse en una meta de reanimación.

Bucheie G, Ospina-Tascon G et al. How to evaluate the microcirculation? Report of a round table conference. Critical Care 2007; 11: R101-9.

9. Boerma EC, Mathura KR, Van Der Voort P, Spronk PB, INCE C ET AL Quantifying bedside-derived imaging of microcirculatory abnormalities in septic patients: a prospective validation study. Crit Care 2005; 9: R601-6.

10. Cornejo R, Downey P, Castro R, Romero C, Reguira T, VEGA J ET AL. High-volume hemofiltration as salvage therapy in severe hyperdynamic septic shock. Intensive Care Med 2006; 32: 713-23.

11. Dewnger R, Carlet J, Masur H, Geriach H, Calandra T ET AL. Surviving sepsis campaing guidelines for management of severe sepsis and septic shock. Crit Care Med 2006; 32: 858-73.

12. Spronk P, Ince C, Gardien M, Mathura K, OudemansVan Straaten H, Zandstra D. Nitroglycerine in septic shock after intravascular volume resuscitation. Lancet 2002; 360: 1395-6.

13. De Backer D, Creteur J, Dubois M J, Sakr Y, Koch M, VERDANT C ET aL. The effects of dobutamine on microcirculatory alterations in patients with septic shock are independent of its systemic effects. Crit Care Med 2006; 34: 403-8.

14. Cariou A, Vinsonneau C, Dhainaut J-F. Adjunctive therapies in sepsis: an evidence-based review. Crit Care Med 2004; (suppl 11): S562-S570. 